\title{
Influence of immersion depth of the sheet pile on the intensity of suffusion processes in pressure hydraulic structures
}

\author{
Iurii Bik $^{1 *}$, Vera Degtiareva ${ }^{1}$, Oksana Pridanova $^{1}$, Aleksandr Kudriashov $^{1}$, Ruslan Dubrovin ${ }^{2}$ \\ ${ }^{1}$ Siberian State University of Water Transport, 33, Schetinkina st., 630099, Novosibirsk, Russia \\ ${ }^{2}$ Admiral Ushakov Maritime State University, 93 Lenin's avenue, Novorossisk, 353924, Russia
}

\begin{abstract}
The research examines the influence of constructive features of a pressure hydraulic structure on the intensity of suffusion processes in the tailwater. The equations of non-vortex filtration fluid motion under a hydraulic structure in the form of a sheet pile wall are calculated by the finite element method using the Neumann and Dirichlet boundary conditions. The influence of immersion depth of the sheet pile on the erosion processes in the tailwater is investigated. The possibility of protecting the pressure head structure by creating an apron in the tailwater which prevents soil suffusion is analyzed. Calculations show that the greatest pressure gradients are observed at the sheet pile tip and at the outlet to the tailwater. From the point of view of the suffusion process, the zone at the lower end of the sheet pile is not dangerous; therefore, predictions about the suffusion intensity for a particular structure should be made on the basis of the output gradients at the tailwater border. Calculations show that the constructive features of a pressure hydraulic structure have a significant impact on the processes of suffusion and pile heave. The graphs are based on the calculation results; they clearly demonstrate that the filtration rate in the structure tailwater sharply decreases with an increase in the depth of driving the sheet pile, regardless of the soil type of the structure base. Besides, an additional protective effect is provided by a structure in the apron tailwater.
\end{abstract}

\section{Introduction}

The abruptly changing pressure movement of groundwater under a hydraulic structure causes a number of negative phenomena that must be taken into account both in the design and in the operation of pressure hydraulic structures. Foremost, significant additional pressure on the structure base which must be considered in the static calculations of the structure arises during the liquid filtration movement under the dam.

Furthermore, filtration can cause significant water losses from the headwater for certain soils of the structure base.

Finally, if the velocities of the liquid filtration movement exceed the permissible values, then erosion in the structure tailwater (suffusion) is possible; as a result, there is a loss of structure stability followed by its displacement in the tailwater direction.

\section{Assessment of possible soil erosion in the structure tailwater depending on the constructive features of the latter}

The factors influencing the intensity of the suffusion processes are investigated in the paper. In this case, the liquid pressure movement in a flat section of the structure is considered. It is known that the steady motion of groundwater is described by the following differential equations $[1,2,3,4,5]$

$$
\begin{aligned}
& u_{x}=-k \frac{\partial H}{\partial x} ; \\
& u_{y}=-k \frac{\partial H}{\partial y} ; \\
& \frac{\partial u_{x}}{\partial x}+\frac{\partial u_{y}}{\partial y}=0 .
\end{aligned}
$$
flow;

where $\mathrm{u}_{\mathrm{x}}$ and $\mathrm{u}_{\mathrm{y}}-$ velocity components of filtration

$\mathrm{k}$ - filtration coefficient corresponding to the structure base soil;

$\mathrm{H}$ - pressure at the design point of the base.

The well-known Laplace equation can be obtained after differentiating the first two equations and substituting the results into the last [1]. It represents the dependence of the fluid pressure in the filtration zone on the calculation point coordinates in differential form

$$
\frac{\partial^{2} H}{\partial x^{2}}+\frac{\partial^{2} H}{\partial y^{2}}=0 \text {. }
$$

\footnotetext{
* Corresponding author: yibik@mail.ru
} 
Equation (2) was solved by the finite difference method owing to discretizing the filtration area in order to determine the pressure at the hydraulic structure base (Figures 1 - 4).

The values of the pressure function $\mathrm{H}(\mathrm{x}, \mathrm{y})$ were determined at each node of the computational grid superimposed on the filtration region. Laplace differential equation was approximated by the corresponding differences to perform calculations by the finite difference method. As a result, equation (2) was transformed to the form

$$
\begin{aligned}
& \frac{H_{m+1, n}-2 H_{m, n}+H_{m-1, n}}{\Delta x^{2}}+ \\
& \frac{H_{m, n+1}-2 H_{m, n}+H_{m, n-1}}{\Delta y^{2}}=0,
\end{aligned}
$$

where $H_{m, n}$ - value of the pressure function imposed on the grid filtration area at the considered node.

The grid dimensions are taken to be the same along the $\mathrm{x}$ and $\mathrm{y}$ axes to simplify calculations, then equation (3) can be written as

$$
\begin{aligned}
& -4 H_{m, n}+H_{m+1, n}+H_{m-1, n}+H_{m, n+1}+ \\
& H_{m, n-1}=0 .
\end{aligned}
$$

Equations (4) were compiled for each node of the calculated filtration area to compute the pressure in the filtration zone.

Two types of boundary conditions were considered in the calculations:

- Dirichlet conditions, according to which the pressure value was set on the bottom line in the headwater and tailwater;

- Neumann conditions, according to which the filtration area and the structure contours bounded by the design grid were considered impenetrable.

\section{Calculation results}

The analysis of the depth influence of driving the sheet pile on the suffusion processes intensity was carried out on the basis of the above calculation methodology.

Equation systems for calculating the filtration process for various structural schemes of a pressure hydraulic structure are given below. The discrete scheme and the outcomes of solving the equations corresponding system are displayed in the diagrams. Calculations are performed in the Mathcad program.

The equation system for calculating the problem of fluid filtration under a sheet pile wall with a pressure in the headwater $\mathrm{H}=10$ meters and in the tailwater $\mathrm{H}=2$ meters with a sheet pile driving depth of 1 meter has the form (Figure 1)

$$
\begin{aligned}
& -4 H_{1,0}+2 H_{1,2}+H_{2,0}+10=0 \\
& -4 H_{2,0}+2 H_{2,1}+2 H_{2,0}=0 \\
& -4 H_{1,2}+H_{1,0}+H_{1,3}+2 H_{2,1}=0 \\
& -4 H_{2,1}+H_{2,0}+H_{2,2}+2 H_{1,2}=0 \\
& -4 H_{1,3}+H_{1,2}+H_{2,2}+2=0 \\
& -4 H_{2,2}+2 H_{2,1}+2 H_{1,3}=0
\end{aligned}
$$

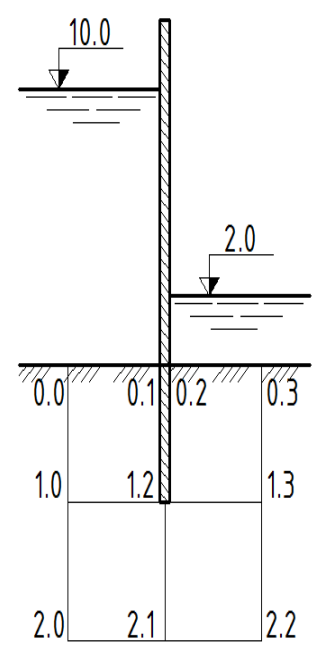

a

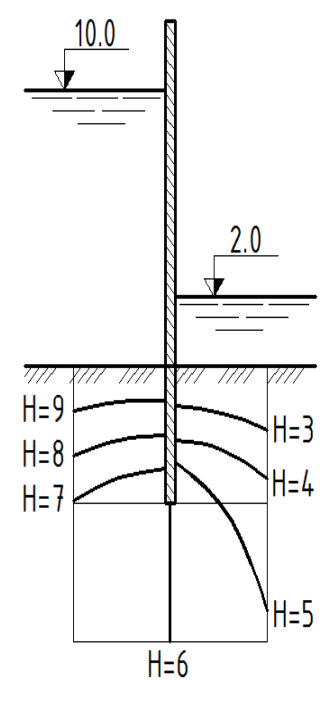

b
Fig.1. Filtration calculation at a sheet pile driving depth of 1 meter:

(a) - discretization of the filtration zone;

(b) - calculation result.

The equation system for calculating the problem of fluid filtration under a sheet pile wall with a pressure in the headwater $\mathrm{H}=10$ meters and in the tailwater $\mathrm{H}=2$ meters with a sheet pile driving depth of 2 meters has the form (Figure 2)

$$
\begin{aligned}
& -4 H_{1,0}+2 H_{1,1}+H_{2,0}+10=0 \\
& -4 H_{1,1}+2 H_{1,0}+H_{2,2}+10=0 \\
& -4 H_{1,2}+2 H_{1,3}+H_{2,2}+2=0 \\
& -4 H_{1,3}+2 H_{1,2}+H_{2,3}+2=0 \\
& -4 H_{2,0}+2 H_{2,2}+H_{3,0}+H_{1,0}=0 \\
& -4 H_{2,2}+H_{2,0}+H_{2,3}+2 H_{3,2}=0 \\
& -4 H_{2,3}+2 H_{2,2}+H_{3,3}+H_{1,3}=0 \\
& -4 H_{3,0}+2 H_{3,2}+2 H_{2,0}=0 \\
& -4 H_{3,2}+H_{3,0}+H_{3,3}+2 H_{2,2}=0 \\
& -4 H_{3,3}+2 H_{3,2}+2 H_{2,3}=0
\end{aligned}
$$




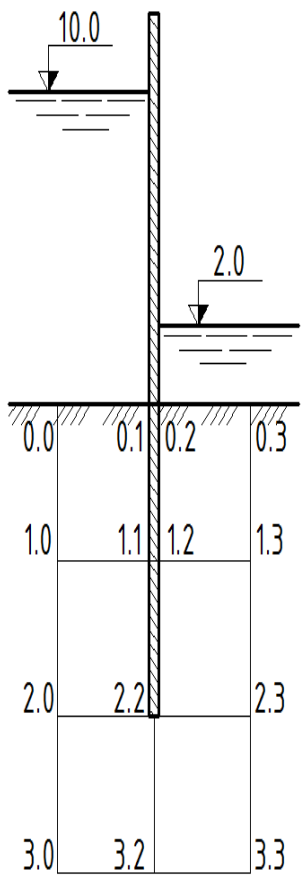

a

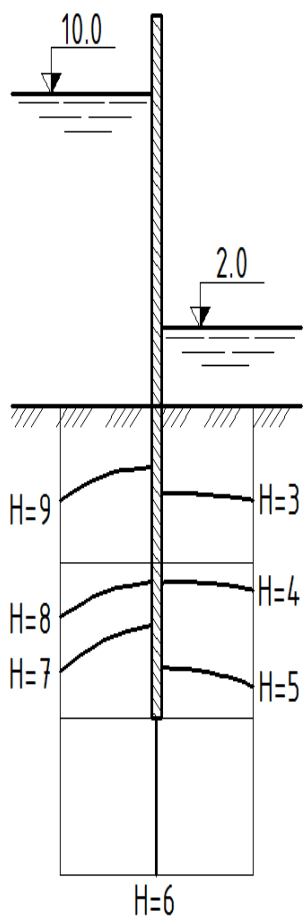

b
Fig. 2. Filtration calculation at a sheet pile driving depth of 2 meters:

(a) - discretization of the filtration zone;

(b) - calculation result.

The equation system for calculating the problem of fluid filtration under a sheet pile wall with a pressure in the headwater $\mathrm{H}=10$ meters and in the tailwater $\mathrm{H}=2$ meters with a sheet pile driving depth of 3 meters has the form (Figure 3)

$$
\begin{aligned}
& -4 H_{1,0}+2 H_{1,1}+H_{2,0}+10=0 \\
& -4 H_{1,1}+2 H_{1,0}+H_{2,1}+10=0 \\
& -4 H_{1,2}+2 H_{1,3}+H_{2,2}+2=0 \\
& -4 H_{1,3}+2 H_{1,2}+H_{2,3}+2=0 \\
& -4 H_{2,0}+2 H_{2,1}+H_{1,0}+H_{3,0}=0 \\
& -4 H_{2,1}+2 H_{2,0}+H_{1,1}+H_{3,2}=0 \\
& -4 H_{2,2}+2 H_{2,3}+H_{1,2}+H_{3,2}=0 \\
& -4 H_{2,3}+2 H_{2,2}+H_{1,3}+H_{3,3}=0 \\
& -4 H_{3,0}+2 H_{3,2}+H_{2,0}+H_{4,0}=0 \\
& -4 H_{3,2}+H_{3,0}+H_{3,3}=2 H_{4,2}=0 \\
& -4 H_{4,0}+2 H_{4,2}+2 H_{3,0}=0 \\
& -4 H_{4,2}+H_{4,0}+H_{4,3}+2 H_{3,2}=0 \\
& -4 H_{4,3}+2 H_{4,2}+2 H_{3,3}=0 \\
& -4 H_{3,3}+2 H_{3,2}+H_{2,3}+H_{4,3}=0
\end{aligned}
$$
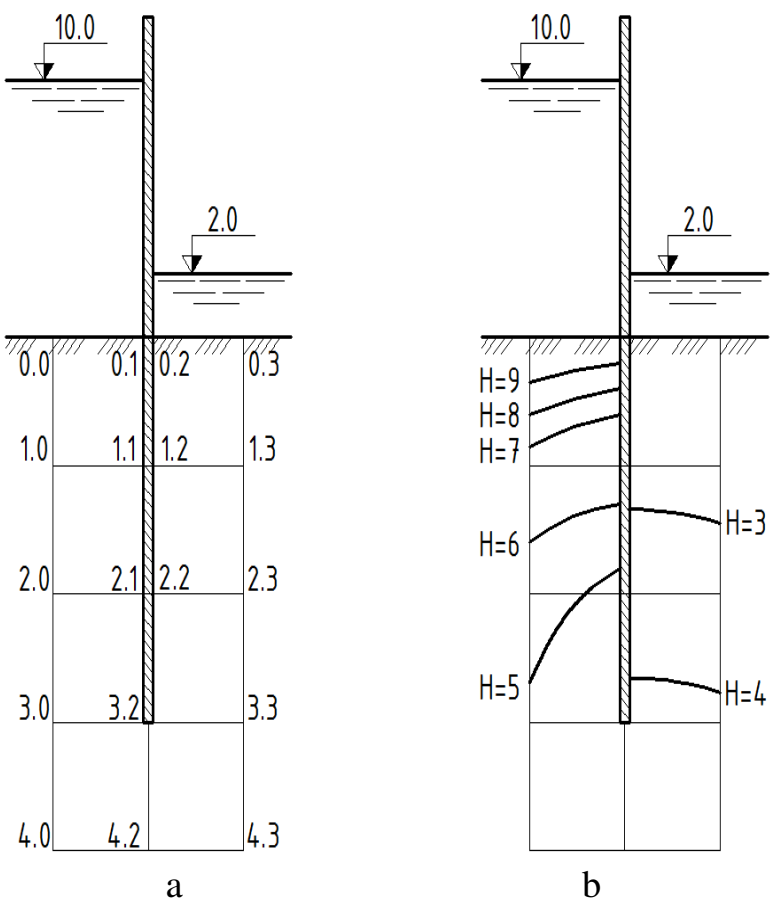

Fig. 3. Filtration calculation at a sheet pile driving depth of 3 meters:

(a) - discretization of the filtration zone;

(b) - calculation result.

Consider the effect of the apron presence in the tailwater structure on the filtration process.

The equation system for calculating the problem of fluid filtration under a sheet pile wall with a pressure in the headwater $\mathrm{H}=10$ meters and in the tailwater $\mathrm{H}=2$ meters with a sheet pile driving depth of 1 meter and the apron device in the tailwater has the form (Figure 4)

$$
\begin{aligned}
& -4 H_{0,1}+4+2 H_{1,1}=0 \\
& -4 H_{1,0}+2 H_{1,1}+H_{2,0}+10=0 \\
& -4 H_{1,1}+H_{1,0}+H_{1,2}+2 H_{2,1}=0 \\
& -4 H_{1,2}+H_{1,1}+H_{1,3}+H_{2,2}+2=0 \\
& -4 H_{1,3}+2 H_{1,2}+H_{2,3}+2=0 \\
& -4 H_{2,0}+2 H_{2,1}+2 H_{1,0}=0 \\
& -4 H_{2,1}+H_{2,0}+H_{2,2}+2 H_{1,1}=0 \\
& -4 H_{2,2}+H_{2,1}+H_{2,3}+2 H_{1,2}=0 \\
& -4 H_{2,3}+2 H_{2,2}+2 H_{1,3}=0
\end{aligned}
$$




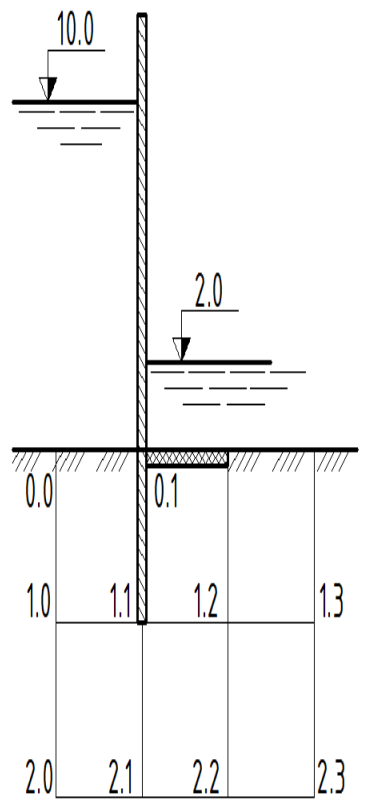

a

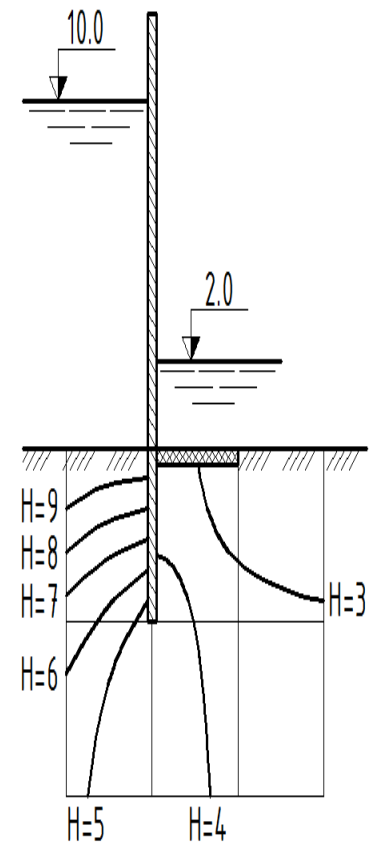

b

Fig. 4. Filtration calculation at a sheet pile driving depth of 1 meter and the apron device in the tailwater structure:

(a) - discretization of the filtration zone;

(b) - calculation result.

As noted above, the integrity of the soil base can be violated as a result of suffusion, soil uplift, contact erosion or contact burst processes under the influence of the filtration fluid movement that occurs under the structure.

The intensity of the suffusion process of soil removal increases significantly with a velocity growth of the filtration flow

$$
u=k J=k\left(H_{n}-H_{n-1}\right) / \Delta n .
$$

where $\mathrm{k}$ - filtration coefficient depending on the soil composing the structure base;

$\mathrm{u}$ - filtration flow rate;

$\mathrm{J}$ - piezometric slope;

$\mathbf{D n}$ - normal distance between pressure lines.

From the earlier performed calculations, the greatest gradients of the filtration flow are observed at the end of the sheet pile and at the outlet to the tailwater. From the point of view of the suffusion process, the zone at the lower end of the sheet pile is not dangerous; therefore, predictions about the possible suffusion for a particular structure should be made on the basis of the output gradients at the tailwater border. Calculations show that the structure constructive features have a significant impact on the suffusion processes (Figure 5,6).

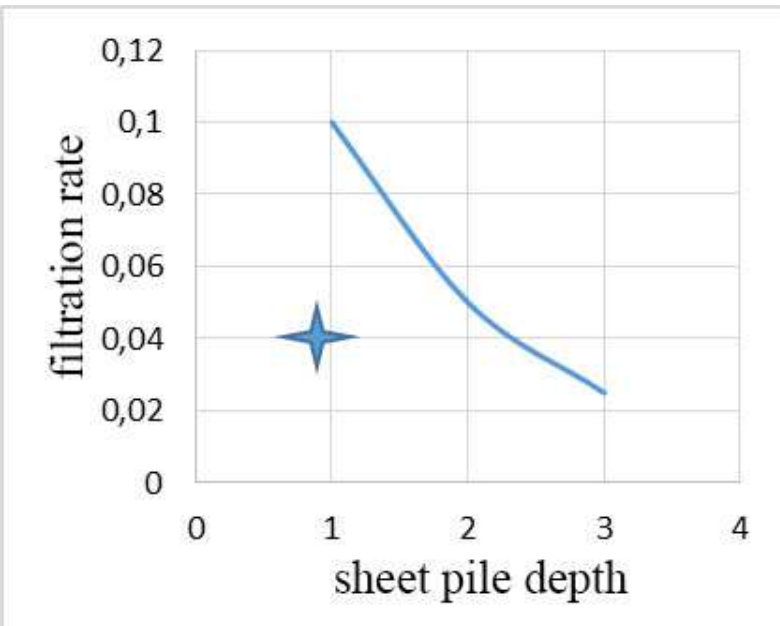

Fig. 5. Dependence of the filtration rate in the structure tailwater on the soil plugging depth for gravel $\left(\mathrm{k}=3,5^{*} 10^{-3}\right)$. The asterisk shows the filtration rate value during the apron structure.

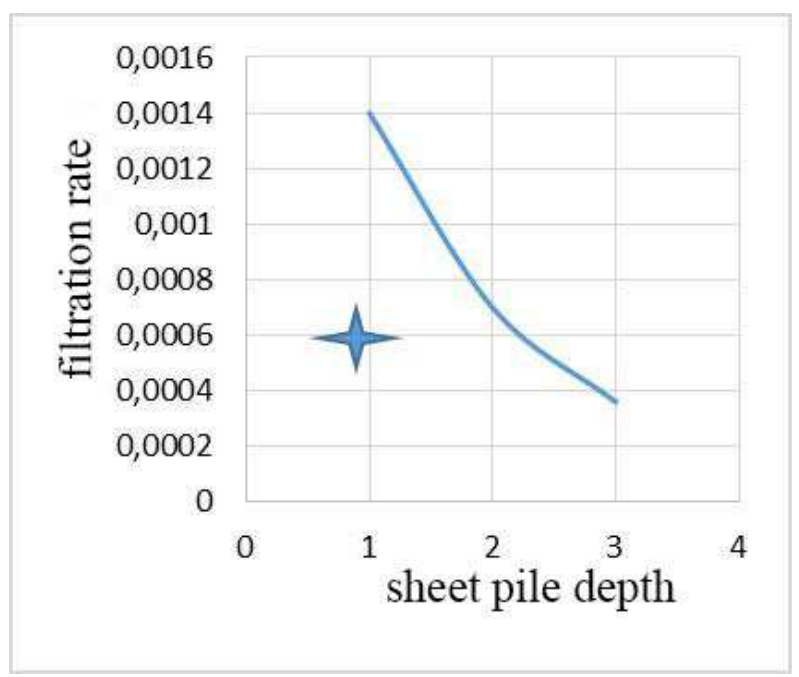

Fig. 6. Dependence of the filtration rate in the structure tailwater on the soil plugging depth for medium-sized sand $\left(\mathrm{k}=5^{*} 10^{-4}\right)$. The asterisk shows the filtration rate value during the apron structure.

\section{Conclusion}

The conducted graphs prove that the filtration rate in the structure tailwater decreases sharply with an increase in the sheet pile depth. The process nature does not depend on the soil type of the structure base.

Besides, the structure in the apron tailwater gives a supplementary essential effect. Note that a flexible asphalt concrete mat can be used as an apron, since this part of the structure does not bear significant loads. Its task is to exclude the possibility of suffusion at the sheet pile base.

The outcomes of the above calculations are best approximated by the following exponential relationships 
to compute the flow filtration rate at the soil boundary in the structure tailwater.

$$
\begin{aligned}
& u=0,2 e^{-0,7 x} \\
& u=0,0027 e^{-0,7 x}
\end{aligned}
$$

The first dependence is drawn up for the gravel base under the structure, the second - for medium-sized sands. The difference in filtration speed is two orders of magnitude.

Based on the performed calculations, it can be concluded that with a driving depth of about 2.2 meters, suffusion in the tailwater is not practically observed when considering the results of experimental studies by V.S. Istomina [1], namely, the permissible piezometric slope, excluding suffusion processes in the tailwater, is about 1.25 with a homogeneous composition of the soil base.

If the soil is significantly heterogeneous, then the permissible piezometric slope should be taken as equal to $0.3-0.4$. Therefore, it is recommended to increase the depth of driving the sheet pile up to 4 meters.

We assess the permissible depth of driving the sheet pile by dint of the possibility of soil uplift under the filtration flow influence. It is possible to obtain dependence for the critical value of the filtration flow gradient, equating the hydrodynamic effect force of the flow per volume unit of soil in the structure tailwater with the force of this soil weight in suspension state.

$$
J_{\kappa p}=\frac{\rho_{2}}{\rho}-(1-n),
$$

where $\rho_{\mathrm{s}}, \rho$-density of soil and water, respectively; $\mathrm{n}-$ soil porosity in relative units.

The calculations indicate that the critical gradient of the filtration flow, computed by formula (7), for medium-sized sand is about 1.1. According to our calculations, the permissible depth of driving the sheet pile will be approximately 2.5 meters (Figure 7).

Thus, the calculations for the soil suffusion opportunity and its uplift in the structure tailwater in the form of a sheet pile wall provide us with practically the same results.

Consequently, it can be said with great confidence that when calculating the possibility of suffusion in the structure tailwater, the required value of the sheet pile driving depth is obtained from the point of view of the structure safe operation. The same value can be taken in order to exclude soil uplift in the computed zone under consideration.

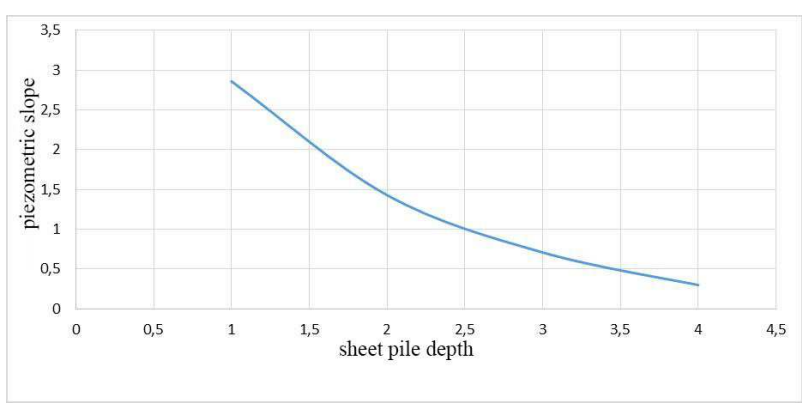

Fig. 7. Dependence of the piezometric slope on the sheet pile depth.

\section{References}

1. D.V. Shterenlicht, Hydraulics (Moscow: Energoatomizdat) (1984)

2. Yu.I. Bik, V.V. Degtyareva. River transport (XXI century). 1 (85), 34-35, (2018).

3. A.S. Bestuzheva, K.K. Anakhaev. Hydrotechnical construction 1, 35-42, (2020)

4. L. C. Van Rijn J. Hydraulic Engineering ACE, 122(9), 867-8746 (1986)

5. A.B. Klaven, Z.D. Kopaliani Experimental studies and hydraulic modeling of river flow and channel processes (SPb: Nestor-History) (2011) 